\title{
A dynamical model for the heavily ram pressure stripped Virgo spiral galaxy NGC 4522 ${ }^{\star}$
}

\author{
B. Vollmer ${ }^{1}$, M. Soida ${ }^{2}$, K. Otmianowska-Mazur ${ }^{2}$, J. D. P. Kenney ${ }^{3}$, J. H. van Gorkom ${ }^{4}$, and R. Beck ${ }^{5}$ \\ 1 CDS, Observatoire astronomique de Strasbourg, 11 rue de l'université, 67000 Strasbourg, France \\ e-mail: bvollmer@astro.u-strasbg.fr \\ 2 Astronomical Observatory, Jagiellonian University, Kraków, Poland \\ 3 Yale University Astronomy Department, PO Box 208101, New Haven, CT 06520-8101, USA \\ 4 Department of Astronomy, Columbia University, 538 West 120th Street, New York, NY 10027, USA \\ 5 Max-Planck-Insitut für Radioastronomie, Auf dem Hügel 69, 53121 Bonn, Germany
}

Received 2 February 2006 / Accepted 27 March 2006

\section{ABSTRACT}

\begin{abstract}
Context. Recent $\mathrm{HI}$ and $\mathrm{H} \alpha$ observations of NGC 4522 have revealed that this spiral galaxy represents one of the best cases of ongoing ram pressure stripping in the Virgo cluster.

Aims. We determined the parameters of the interaction between the interstellar medium of NGC 4522 and the intracluster medium of the Virgo cluster.

Methods. A dynamical model including ram pressure stripping is applied to the strongly HI deficient Virgo spiral galaxy NGC 4522. A carefully chosen model snapshot is compared with existing VLA HI observations.

Results. The model successfully reproduces the large-scale gas distribution and the velocity field. However it fails to reproduce the large observed HI linewidths in the extraplanar component, for which we give possible explanations. In a second step, we solve the induction equation on the velocity fields of the dynamical model and calculate the large scale magnetic field. Assuming a Gaussian distribution of relativistic electrons we obtain the distribution of polarized radio continuum emission which is also compared with our VLA observations at $6 \mathrm{~cm}$. The observed maximum of the polarized radio continuum emission is successfully reproduced. Our model suggests that the ram pressure maximum occurred only $~ 50 \mathrm{Myr}$ ago.

Conclusions. Since NGC 4522 is located far away from the cluster center $(\sim 1 \mathrm{Mpc})$ where the intracluster medium density is too low to cause the observed stripping if the intracluster medium is static and smooth, two scenarios are envisaged: (i) the galaxy moves very rapidly within the intracluster medium and is not even bound to the cluster; in this case the galaxy has just passed the region of highest intracluster medium density; (ii) the intracluster medium is not static but moving due to the infall of the M 49 group of galaxies. In this case the galaxy has just passed the region of highest intracluster medium velocity. This study shows the strength of combining high resolution $\mathrm{HI}$ and polarized radio continuum emission with detailed numerical modeling of the evolution of the gas and the large-scale magnetic field.
\end{abstract}

Key words. galaxies: individual: NGC 4522 - galaxies: interactions - galaxies: kinematics and dynamics - galaxies: ISM

\section{Introduction}

The spiral galaxy NGC 4522 is one of the best examples for ongoing ram pressure stripping due to the galaxy's rapid motion within the hot and tenuous intracluster gas (ICM) of the Virgo cluster. HI and $\mathrm{H} \alpha$ observations (Kenney et al. 2004; Kenney \& Koopmann 1999) showed a heavily truncated gas disk at a radius of $3 \mathrm{kpc}$, which is $\sim 40 \%$ of the optical radius, and a significant amount of extraplanar gas to the west of the galactic disk. The one-sided extraplanar atomic gas distribution shows high column densities, comparable to those of the adjectant galactic disk. Such high extraplanar gas column densities are unusual among Virgo spiral galaxies (Cayatte et al. 1990), suggesting that NGC 4522 is in a short-lived phase of evolution.

Since the stellar disk is symmetric and undisturbed (Kenney \& Koopmann 1999), a tidal interaction is excluded as the origin of the peculiar gas distribution of NGC 4522. A scenario where NGC 4522 is moving roughly to the east and experiences strong ram pressure can account for the truncated gas disk and

* A movie is only available in electronic form at http://www.edpsciences.org the western extraplanar gas. A scenario where the ram pressure peak occurred a few 100 Myr ago (stripping by the intracluster medium around M 87; Vollmer et al. 2000) is excluded, because the HI column density distribution and velocity field are inconsistent with fall back of stripped gas which should occur a few $100 \mathrm{Myr}$ after peak ram pressure, which is needed to create extraplanar gas at these late times. Further evidence for the peak ram pressure scenario comes from polarized radio continuum observations (Vollmer et al. 2004). The $6 \mathrm{~cm}$ polarized emission is located at the eastern edge of the galactic disk, opposite to the western extraplanar gas. This ridge of polarized radio continuum emission is most likely due to ram pressure compression of the interstellar medium (ISM) and its magnetic field. In addition, the degree of polarization decreases from the east to the west and the flattest spectral index between $20 \mathrm{~cm}$ and $6 \mathrm{~cm}$ coincides with the peak of the $6 \mathrm{~cm}$ polarized emission. These findings are also consistent with a scenario where ram pressure is close to its maximum.

This scenario has one important caveat: NGC 4522 is located at a projected distance of $\sim 1 \mathrm{Mpc}$ from the center of the Virgo cluster (M 87). Assuming a static smooth ICM and standard 
Table 1. Total mass, number of particles $N$, particle mass $M$, and smoothing length $l$ for the different galactic components.

\begin{tabular}{lllll}
\hline \hline component & $M_{\text {tot }}\left(M_{\odot}\right)$ & $N$ & $M\left(M_{\odot}\right)$ & $l(\mathrm{pc})$ \\
\hline halo & $4.8 \times 10^{10}$ & 32768 & $1.48 \times 10^{6}$ & 1200 \\
bulge & $1.7 \times 10^{9}$ & 16384 & $1.0 \times 10^{5}$ & 180 \\
disk & $8.3 \times 10^{9}$ & 32768 & $2.5 \times 10^{5}$ & 240 \\
\hline
\end{tabular}

values for the ICM density and the galaxy velocity, the ram pressure at that location seems to be too low by an order of magnitude to produce the observed truncation of the gas disk. Therefore, Kenney et al. (2004) and Vollmer et al. (2004) claim that either (i) NGC 4522 has a velocity of $\sim 4000 \mathrm{~km} \mathrm{~s}^{-1}$ with respect to the Virgo cluster mean or (ii) that the ICM density is locally enhanced or (iii) that the intracluster medium is moving or (iv) a combination of (ii) and (iii). Based on existing X-ray observations Kenney et al. (2004) claim that the latter scenario is more likely, given that NGC 4522 is located between M 87 and the bright elliptical galaxy M 49 which is the center of a galaxy subcluster falling into the Virgo cluster from behind (Irwin \& Sarazin et al. 1996 and Biller et al. 2004). In addition, the X-ray hotspot detected by Shibata et al. (2001) represents the sign of an interaction between the Virgo intracluster medium and that of the M 49 subcluster. The intracluster medium of the M49 subcluster has thus a velocity whose radial component is opposite to the radial velocity of NGC 4522 . This moving intracluster medium together with a modest local density enhancement of the intracluster medium by a factor of 2-4 can account for the observed stripping radius.

In this article we present a ram pressure stripping model for NGC 4522 in order to assess if a simulation using a time dependent ram pressure can account for the observed gas distribution, velocity field, and polarized radio continuum emission distribution. Our aim is to constrain the parameters of the event. Since the observations of Kenney et al. (2004) and Vollmer et al. (2004) showed that ram pressure is ongoing, we only consider model snapshots close to peak ram pressure.

In Sect. 2 we describe the dynamical model. The choice of the best fit model is justified and its time evolution and the final snapshot for the comparison with observations is presented in Sect. 3. The comparison with VLA HI data is done using moment maps and position-velocity diagrams (Sect. 4). We solved the induction equation for the velocity field of our simulation to obtain the evolution of the large scale magnetic field and the distribution of the polarized radio continuum emission which is compared with observations in Sect. 5. We discuss our results in Sect. 6 and give our conclusions in Sect. 7 .

\section{Dynamical model}

We use the $N$-body code described in Vollmer et al. (2001) which consists of two components: a non-collisional component that simulates the stellar bulge/disk and the dark halo, and a collisional component that simulates the ISM.

The non-collisional component consists of 49125 particles, which simulate the galactic halo, bulge, and disk. The characteristics of the different galactic components are shown in Table 1. The resulting rotation velocity is $\sim 100 \mathrm{~km} \mathrm{~s}^{-1}$ and the rotation curve becomes flat at a radius of about $4 \mathrm{kpc}$.

We have adopted a model where the ISM is simulated as a collisional component, i.e. as discrete particles which possess a mass and a radius and which can have inelastic collisions (sticky particles). Since the ISM is a turbulent and fractal medium (see e.g. Elmegreen \& Falgarone 1996), it is neither continuous nor discrete. The volume filling factor of the warm and cold phases is smaller than one. The warm neutral and ionized gas fill about $30-50 \%$ of the volume, whereas cold neutral gas has a volume filling factor smaller than $10 \%$ (Boulares \& Cox 1990). It is not clear how this fraction changes, when an external pressure is applied. In contrast to smoothed particles hydrodynamics (SPH), which is a quasi continuous approach and where the particles cannot penetrate each other, our approach allows a finite penetration length, which is given by the mass-radius relation of the particles. Both methods have their advantages and their limits. The advantage of our approach is that ram pressure can be included easily as an additional acceleration on particles that are not protected by other particles (see Vollmer et al. 2001). In this way we avoid the problem of treating the huge density contrast between the ICM $\left(n \sim 10^{-4} \mathrm{~cm}^{-3}\right)$ and the ISM $\left(n>1 \mathrm{~cm}^{-3}\right)$ of the galaxy.

The 20000 particles of the collisional component represent gas cloud complexes which are evolving in the gravitational potential of the galaxy. The total assumed gas mass is $M_{\mathrm{gas}}^{\mathrm{tot}}=1.3 \times 10^{9} M_{\odot}$, which corresponds to the total neutral gas mass before stripping, i.e. to an HI deficiency of 0 , which is defined as the logarithm of the ratio between the HI content of a field galaxy of same morphological type and diameter and the observed HI mass. To each particle a radius is attributed depending on its mass. During the disk evolution the particles can have inelastic collisions, the outcome of which (coalescence, mass exchange, or fragmentation) is simplified following Wiegel (1994). This results in an effective gas viscosity in the disk.

As the galaxy moves through the ICM, its clouds are accelerated by ram pressure. Within the galaxy's inertial system its clouds are exposed to a wind coming from a direction opposite to that of the galaxy's motion through the ICM. The temporal ram pressure profile has the form of a Lorentzian, which is realistic for galaxies on highly eccentric orbits within the Virgo cluster (Vollmer et al. 2001). The effect of ram pressure on the clouds is simulated by an additional force on the clouds in the wind direction. Only clouds which are not protected by other clouds against the wind are affected.

The particle trajectories are integrated using an adaptive timestep for each particle. This method is described in Springel et al. (2001). The following criterion for an individual timestep is applied:

$\Delta t_{i}=\frac{20 \mathrm{~km} \mathrm{~s}^{-1}}{a_{i}}$,

where $a_{i}$ is the acceleration of the particle $i$. The minimum value of $t_{i}$ defines the global timestep used for the Burlisch-Stoer integrator that integrates the collisional component.

\section{Search for the best fit model}

In this section we will constrain the parameters of the ram pressure stripping event which are (i) the peak ram pressure; (ii) the temporal ram pressure profile; (iii) time since peak ram pressure; (iv) the inclination angle between the galaxy's disk and the intracluster medium wind direction; and (v) the azimuthal viewing angle for the observed inclination and position angles. These parameters are related to the observed quantities which are (1) the position angle; (2) the inclination angle of the galactic disk; (3) the line-of-sight velocity of the galaxy with respect to the cluster mean; and (4) the projected ICM wind direction. The position angle and inclination of NGC 4522 define a plane in 
three dimensional space. The model galaxy can then be rotated within this plane by the azimuthal viewing angle (see below). The three dimensional model wind direction, the line-of-sight velocity of the galaxy, and the projected ICM wind direction are thus functions of the azimuthal viewing angle.

In the case of a smooth static ICM ram pressure is proportional to the ICM density $\rho_{\text {ICM }}$ and the square of the galaxy velocity with respect to the Virgo cluster $\boldsymbol{v}_{\text {gal }}$. If the ICM is moving with respect to the cluster mean velocity the expression for ram pressure yields:

$p_{\text {ram }}=\rho_{\text {ICM }}\left(\boldsymbol{v}_{\text {gal }}-\boldsymbol{v}_{\mathrm{ICM}}\right)^{2}$,

where $\boldsymbol{v}_{\mathrm{ICM}}$ is the galaxy's velocity vector with respect to the Virgo cluster, and $\boldsymbol{v}_{\text {gal }}$ is the galaxy velocity with respect to the Virgo cluster.

As noted in Kenney et al. (2004) the orbit of NGC 4522 is not easy to understand. Its high radial velocity $\left(\sim 1300 \mathrm{~km} \mathrm{~s}^{-1}\right)$ with respect to the cluster mean velocity excludes a classical radial orbit. At its projected distance from the cluster center $\left(3.3^{\circ} \sim 1 \mathrm{Mpc}\right)$ the density of the intracluster medium is an order of magnitude too low to strip the galaxy's ISM up to a galactic radius of $3 \mathrm{kpc}$ if one assumes a velocity of the galaxy of $1500 \mathrm{~km} \mathrm{~s}^{-1}$ within the cluster (Kenney et al. 2004). Kenney et al. (2004) and Vollmer et al. (2004) propose two possible scenarios enhancing the ram pressure by a factor of 10: (i) the ICM is moving opposite to the trajectory of NGC 4522 due to the infall of the M 49 group into the Virgo cluster and (ii) NGC 4522 is not bound to the cluster and crosses it with a velocity of $\sim 4000 \mathrm{~km} \mathrm{~s}^{-1}$ only once. In both cases we expect a strongly peaked temporal ram pressure profile similar to that for radial orbits:

$p_{\text {ram }}=p_{\text {max }} \frac{t_{\mathrm{HW}}^{2}}{t^{2}+t_{\mathrm{HW}}^{2}}$,

where $t_{\mathrm{HW}}$ is the width of the profile (Vollmer et al. 2001). Since the observations of Kenney et al. (2004) and Vollmer et al. (2004) suggest that ram pressure is close to maximum, we only consider model snapshots close to peak ram pressure $p_{\max }$, which has to be at least $1000 \mathrm{~cm}^{-3}\left(\mathrm{~km} \mathrm{~s}^{-1}\right)^{2}$ to strip gas with a column density of $10 M_{\odot} \mathrm{pc}^{-2}$ at the observed stripping radius of $3 \mathrm{kpc}$ (Kenney et al. 2004). We do not consider higher ram pressure maxima, because this value is already a factor 10 above the estimate based on a static smooth intracluster medium and a velocity of $\sim 1000 \mathrm{~km} \mathrm{~s}^{-1}$. The Gaussian half width $t_{\mathrm{HW}}$ is not critical as long as it is not too large. Since the velocity of NGC 4522 with respect to the intracluster medium (flowing or not) is high, a value between 50 and $100 \mathrm{Myr}$ is reasonable. Thus, we set $p_{\max }=2000 \mathrm{~cm}^{-3}\left(\mathrm{~km} \mathrm{~s}^{-1}\right)^{2}$ and $t_{\mathrm{HW}}=80 \mathrm{Myr}$. We define $t=0 \mathrm{Myr}$ as the time when ram pressure is maximum.

The ram pressure efficiency also depends on the inclination angle $i$ between the galactic disk and the ICM wind direction (Vollmer et al. 2001). Since the HI observations indicate that stripping occurs more face-on, we do not consider edgeon stripping. We made 3 simulations with 3 different inclination angles between the galaxy's disk and the ICM wind direction: (i) $i=45^{\circ}$, (ii) $i=60^{\circ}$, and (iii) $i=75^{\circ}$. An inclination of $i=0^{\circ}$ means that the galactic disk is parallel to the ICM wind direction.

The last free parameter, the azimuthal viewing angle, is chosen in a way to fit the observed HI distribution and to reproduce the positive line-of-sight component of the wind direction (the galaxy is moving away from the observer). In Figs. 1 we show the components of the 3D ICM wind direction (left panels) and

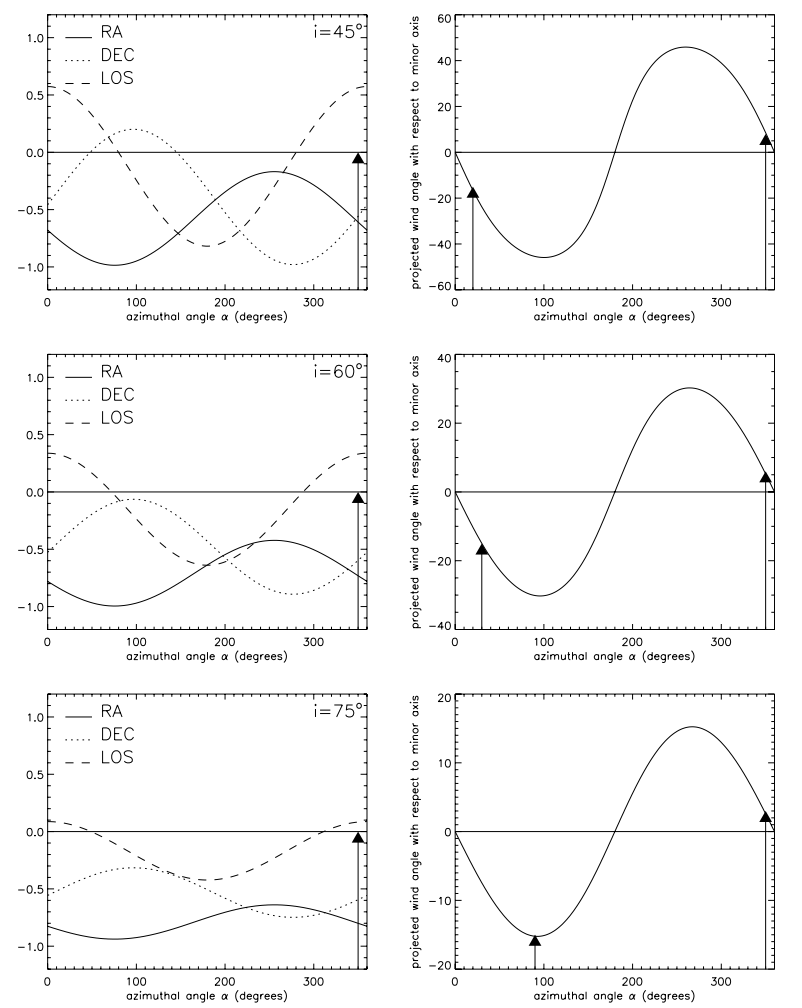

Fig. 1. Inclination angle between the galactic disk and the ICM wind $i=45^{\circ}, i=60^{\circ}$, and $i=75^{\circ}$. Left panels: components of the 3D ICM wind direction as a function of the azimuthal viewing angle $\alpha$. Right panels: projected wind direction with respect to the galaxy's minor axis (counted counter-clockwise) as a function of $\alpha$. The arrows indicate the best fit model $\alpha=350^{\circ}$ and the azimuthal viewing angle for a projected wind angle of $-15^{\circ}$.

the projected wind direction as a function of the azimuthal viewing angle $\alpha$ (right panels). The maximum of the line-of sight component of the ICM wind is largest for $i=45^{\circ}$ and smallest for $i=75^{\circ}$. The wind components along the right ascension and declination are all negative, i.e. the ICM wind blows from the south east. Whereas the two components are roughly equal for $i=45^{\circ}$ and $i=60^{\circ}$, the RA components is smaller than the DEC components for $i=75^{\circ}$. As the maximum of the line-of sight component the maximum of the projected wind direction also varies with $i$. It is maximum for $i=45^{\circ}$ and minimum for $i=75^{\circ}$. In all cases, an azimuthal viewing angle of $\alpha=350^{\circ}$ leads to a positive projected wind direction smaller than $6^{\circ}$. However, if one assumes a given projected wind direction of $-15^{\circ}$, the corresponding azimuthal viewing angles vary: $\alpha\left(45^{\circ}\right)=20^{\circ}, \alpha\left(60^{\circ}\right)=30^{\circ}, \alpha\left(75^{\circ}\right)=90^{\circ}$.

None of the simulations shows significant extraplanar gas before $t=0$ Myr. In Fig. 2 we show 3 timesteps 0 Myr, 50 Myr, and $100 \mathrm{Myr}$ after the ram pressure maximum. The simulation with an inclination angle between the galaxy's disk and the ICM wind direction of $i=45^{\circ}$ does not lead to the observed stripping radius neither shows significant extraplanar gas. The simulation with $i=75^{\circ}$ (Figs. $2 \mathrm{a}-\mathrm{c}$ ) leads to the correct stripping radius and shows extraplanar gas at $t=50 \mathrm{Myr}$ (Fig. 2h) and $t=100 \mathrm{Myr}$ (Fig. 2i). However, the column density of this extraplanar gas is smaller compared to the HI observations. In particular, the observed detached south western extraplanar HI maximum is missing. Only the simulation with $i=60^{\circ}$ at $t=50 \mathrm{Myr}$ (Fig. 2e) can reproduce the observed HI distribution. About 50 Myr later the column density of the northern extraplanar gas has already 

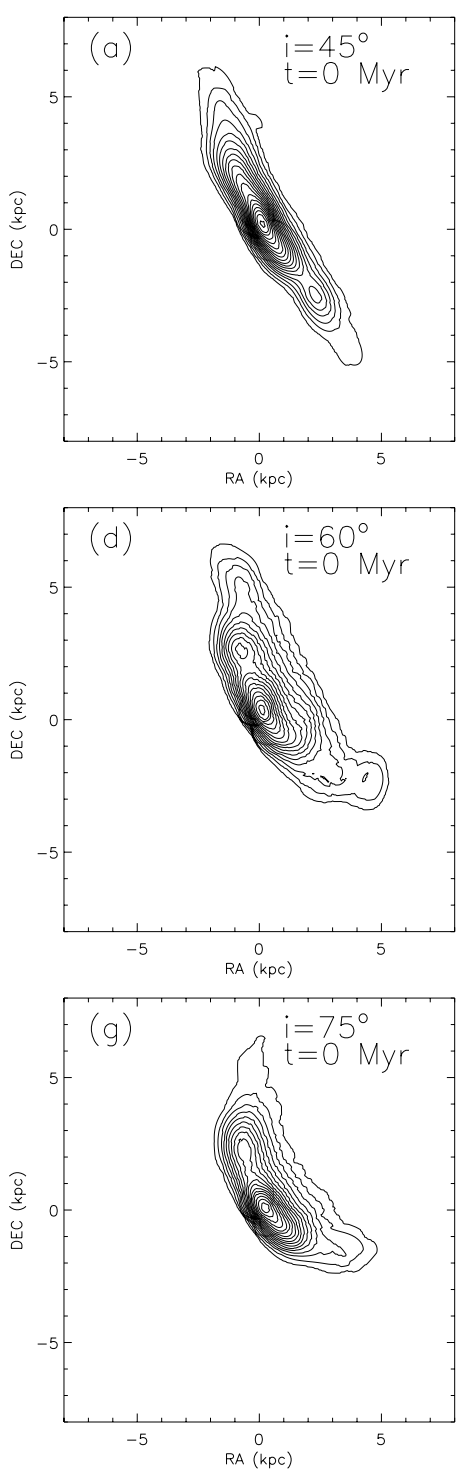
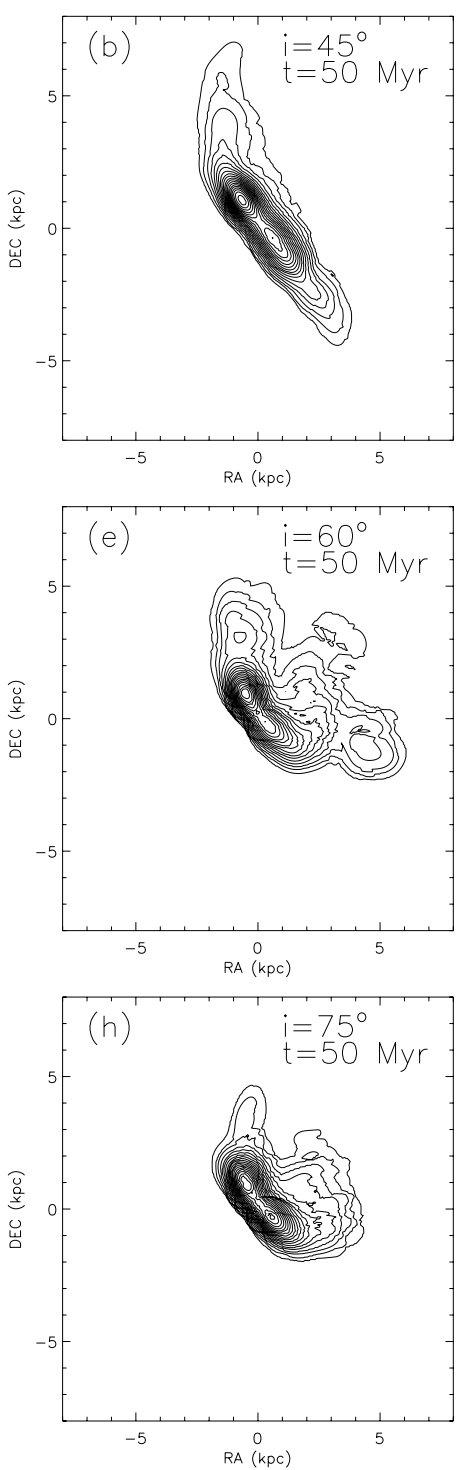
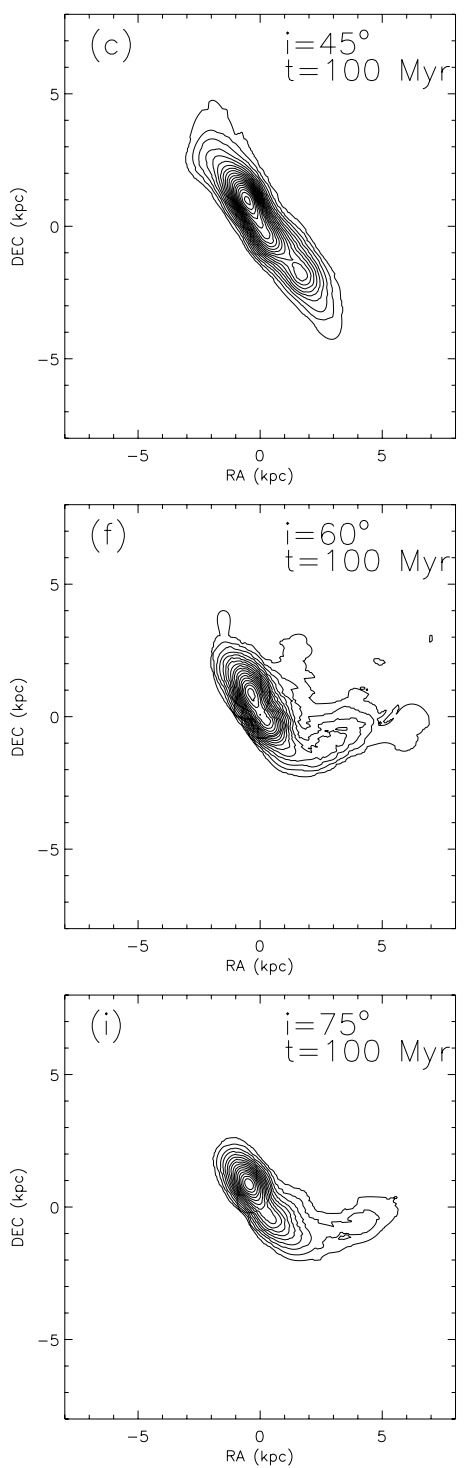

Fig. 2. Model snapshots for different simulations. The ram pressure profile for all simulations is given by Eq. (3). The inclination angle $i$ between the orbital and the galaxy's disk plane and the timestep of the snapshot are varied. Left column: $t=0$ Myr. Middle column: $t=50$ Myr. Right column: $t=100$ Myr. Upper row: $i=45^{\circ}$. Middle row: $i=60^{\circ}$. Lower row: $i=75^{\circ}$.

decreased significantly and the shape of the southern extraplanar gas has changed (Fig. 2f).

As a conclusion, we rule out the $i=75^{\circ}$ simulations, because the galaxy's small line-of-sight velocity component (Fig. 1) would lead to galaxy and/or ICM velocities which are beyond any reasonable values $\left(>4000 \mathrm{~km} \mathrm{~s}^{-1}\right)$. We also rule out the $i=45^{\circ}$ simulations, because it can not reproduce the observed extraplanar gas. We are thus left with the $i=60^{\circ}$ simulation. Recent HST observations of NGC 4522 (Kenney et al., in prep.) yield a projected wind direction of $\sim-15^{\circ}$. The corresponding azimuthal viewing angle is $\alpha=30^{\circ}$. Using this $\alpha$ we realized that the observations can be best reproduced with $t=40 \mathrm{Myr}$ instead of $t=50 \mathrm{Myr}$ used before (Fig. 3). Whereas the HI gas distribution is reproduced in a satisfactory way, the velocity isocontours of the western extraplanar gas are different from the observed ones (Fig. 8).

As an additional test we approximated the temporal ram pressure profile with a step function, i.e. the galaxy is moving abruptly into a region of high intracluster medium density and/or velocity. The step occurs at $t=0 \mathrm{Myr}$ and the subsequent ram
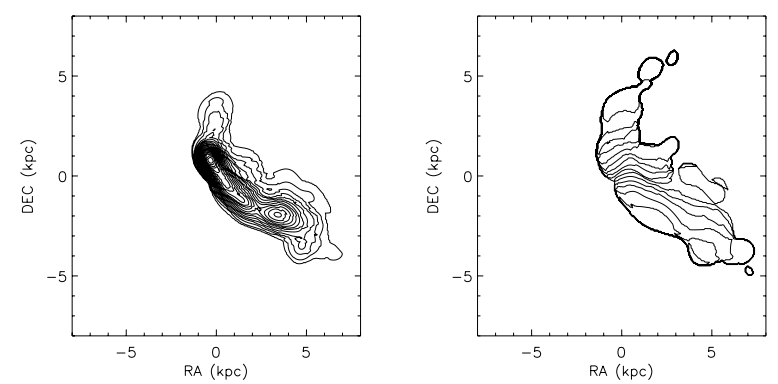

Fig. 3. Snapshot at $t=40 \mathrm{Myr}$ of the simulation with $i=60^{\circ}$. The azimuthal viewing angle is $\alpha=30^{\circ}$. Left panel: gas distribution. Right panel: velocity field.

pressure is assumed to be constant at a level of the maximum ram pressure of the Lorentzian (Eq. (3)). Figure 4 shows the ISM evolution for $i=60^{\circ}$. None of the timesteps show the observed distribution of the extraplanar high column density gas (Fig. 8). We therefore exclude a constant ongoing ram pressure. 

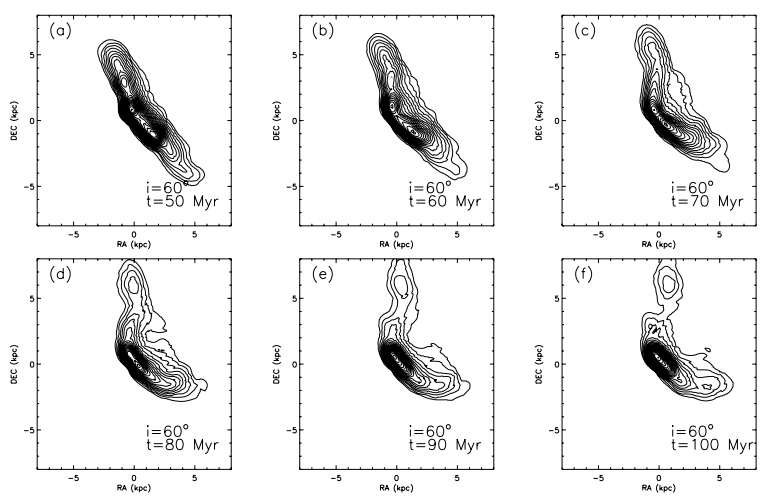

Fig. 4. Snapshots of the simulation using a step function for the temporal ram pressure profile with $i=60^{\circ}$. The azimuthal viewing angle is $\alpha=350^{\circ}$.

Therefore, our best fitting model has the following parameters (see Eq. (3)): $p_{\max }=2000 \mathrm{~cm}^{-3}\left(\mathrm{~km} \mathrm{~s}^{-1}\right)^{2}, t_{\mathrm{HW}}=80 \mathrm{Myr}$, $i=60^{\circ}$, and $t=50 \mathrm{Myr}$. The associated uncertainties are $\Delta i \sim 10^{\circ}, \Delta \alpha \sim 20^{\circ}$, and $\Delta t \sim 10 \mathrm{Myr}$. Subsequently, the radial velocity of NGC 4522 represents $33 \%$ of its total velocity within a static ICM. The total velocity of NGC 4522 with respect to a static ICM is thus $v_{\mathrm{N} 4522} \sim 3500 \mathrm{~km} \mathrm{~s}^{-1}$. Together with a local ICM density of $n_{\mathrm{ICM}}=10^{-4} \mathrm{~km} \mathrm{~s}^{-1}$ this is consistent with our assumed peak ram pressure of $2000 \mathrm{~cm}^{-3}\left(\mathrm{~km} \mathrm{~s}^{-1}\right)^{2}$ within a factor of 1.6.

The large scale evolution of this NGC 4522 simulation can be seen in Fig. 5. The ICM wind begins to drive out the ISM of NGC 4522 only at $-100 \mathrm{Myr}$. The gas is expelled to the west. At the first stage of stripping ( $t \sim-30 \mathrm{Myr})$ the extraplanar gas of highest surface density is found in the south-west. At $t \sim 10 \mathrm{Myr}$ both, the south-western and north-western parts of the extraplanar gas, show a relatively high column density. After the ram pressure maximum has occurred $(t=0 \mathrm{Myr})$ the surface density of the extraplanar gas west to the galaxy center has significantly increased.

According to these simulations we observe the galaxy $\sim 50 \mathrm{Myr}$ after the ram pressure maximum. In the two different scenarios described above this means that (i) the galaxy encountered the intracluster medium of highest density 50 Myr ago or (ii) the galaxy just leaves the region of maximum ICM velocity. The final gas distribution is shown in Fig. 6. The extraplanar gas has a complex asymmetric three-dimensional structure. A part of the ISM initially located at galactic radii between 3 and $5 \mathrm{kpc}$ is stripped in a ring-like configuration, located up to $\sim 4 \mathrm{kpc}$ above the stellar disk.

\section{Comparison with VLA HI observations}

In this section the model snapshot of Fig. 6 is compared with the VLA HI observations of Kenney et al. (2004). For this purpose we assume that only gas with a volume density greater than $\sim 10 \mathrm{~cm}^{-3}$ is in form of neutral hydrogen. This corresponds to the high end of the densities of the warm ionized medium (Boulares \& Cox 1990). The lower density gas is assumed to be ionized. The comparison between the model and observations is done based on moment maps (Sect. 4.1) and a position-velocity diagram (Sect. 4.2).

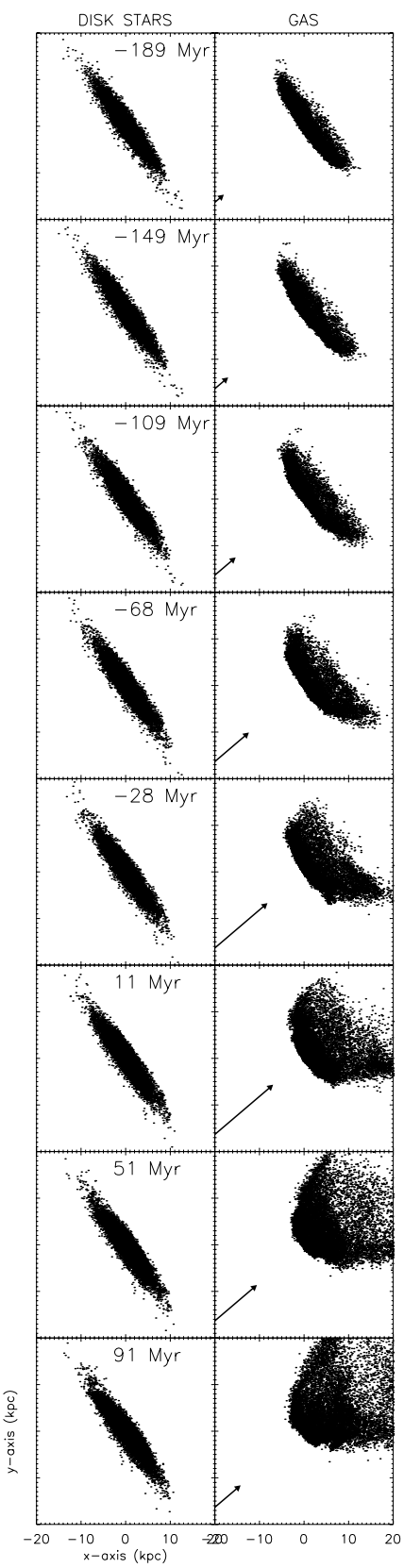

Fig. 5. Evolution of the model stellar (1st column) and gas disk (2nd column). The major axis position angle and inclination of NGC 4522 are $\mathrm{PA}=214^{\circ}$ and $i=80^{\circ}$, respectively. The arrow indicates the direction of ram pressure, i.e. it is opposite to the galaxy's velocity vector, and its size is proportional to $\rho v_{\mathrm{gal}}^{2}$. Maximum ram pressure occurs at $t=0 \mathrm{Myr}$. The timestep of each snapshot is marked in each panel showing the stellar disk.

\subsection{The moment maps}

Since ram pressure selectively affects the gas, the model and observed stellar disks are symmetric whereas the model and observed gas distributions are highly asymmetric (Fig. 7), i.e. the ISM is pushed to the west of the galactic disk.

The HI distribution (moment 0 ), velocity field (moment 1 ), and velocity dispersion (moment 2 ) are shown in Fig. 8. The following observed properties of the HI data are reproduced:

- the model HI deficiency is 0.5 compared to the observed value of $0.6 \pm 0.2$;

- the gas disk is truncated at a radius of about $3 \mathrm{kpc}$; 

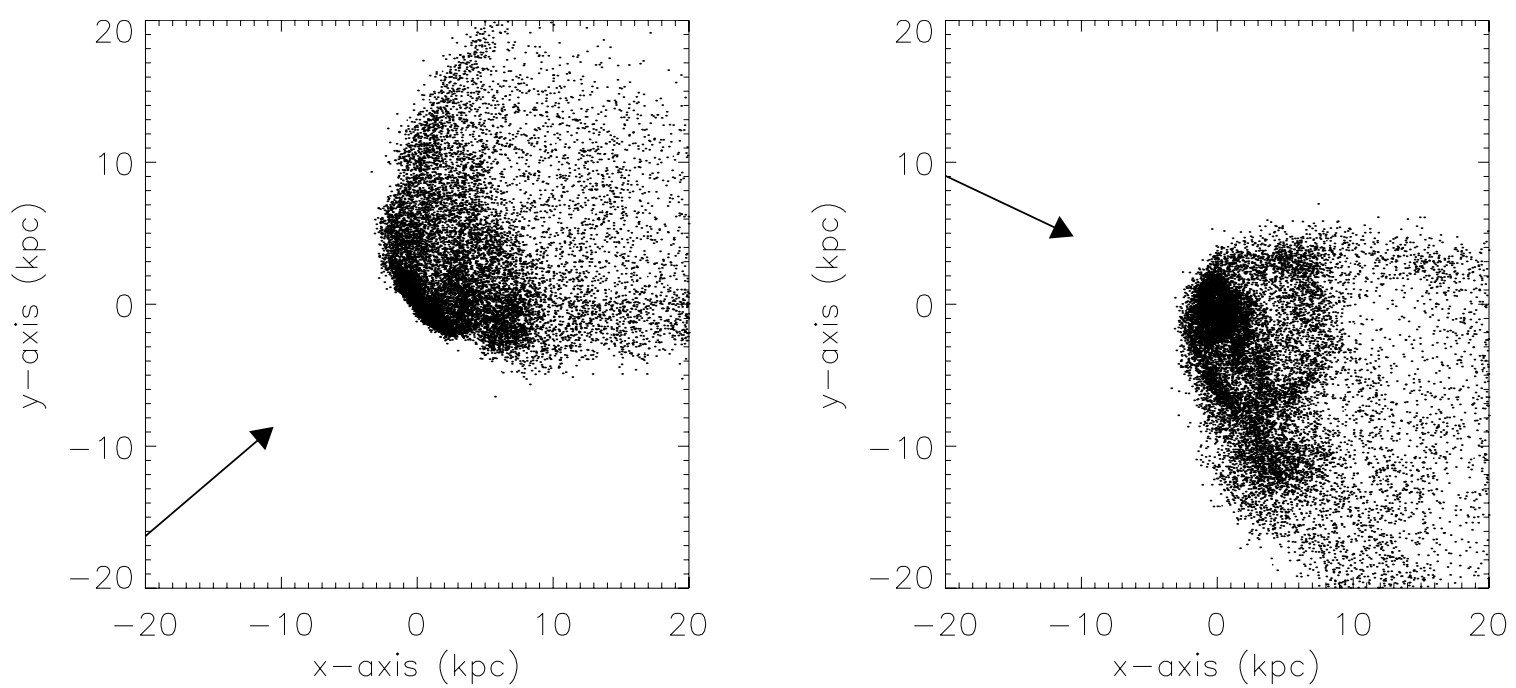

Fig. 6. Comparison snapshot of the gas distribution of NGC 4522. Left panel: view of the disk using the observed position angle and inclination of NGC 4522. Right panel: the disk seen face-on. The arrow indicates the direction of the wind, i.e. opposite to the galaxy's motion.

MODEL

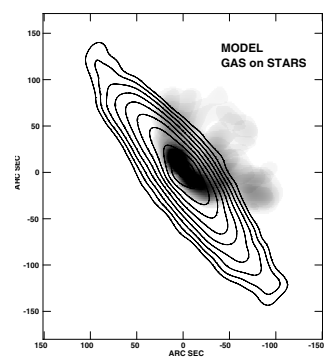

OBSERVATIONS

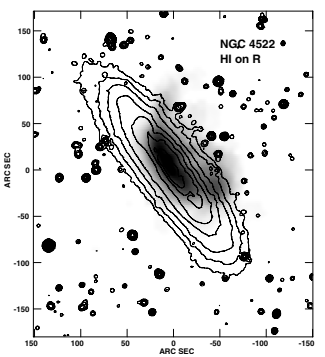

Fig. 7. Stellar (contours) and gas (greyscale) distribution of NGC 4522. Left panel: model. Right panel: HI observations (Kenney et al. 2004).

- within the disk more gas is found in the northern part than in the southern part;

- the existence of a high surface density extraplanar gas component to the west of the galactic disk;

- the extraplanar gas distribution shows two maxima; the south-western maximum is more prominent than the northwestern one;

- the velocity field of the extraplanar gas is relatively regular;

- the extraplanar gas has less extreme velocities than the nearby disk emission, i.e. the velocity contours curve away from the minor axis.

Thus, the overall agreement between the model and observed first two moments (gas distribution and velocity field) is satisfactory. Whereas $40 \%$ of the observed HI mass resides in the extraplanar component, the model yields a fraction of $65 \%$. This difference is likely due to the unknown difference between the initial gas distribution of the model and/or due to the fact that we detect only a fraction of the stripped gas due to ISM expansion, heating and ionization.

The model and observed velocity dispersions are different. In the observations, the peak HI dispersion is not at the nucleus, but in the extraplanar gas, $\sim 30^{\prime \prime}$ west of the nucleus. Even within the disk, the maximum in the observed dispersion is offset from the nucleus by $\sim 10^{\prime \prime}$. Our model shows a maximum in the center, a region of marginally larger linewidth to the north, and a region of larger linewidth within the south western extraplanar gas. The central model peak is due to the model rotation curve which is somewhat steeper than the observed rotation curve. Thus, although we find a small enhancement of the linewidth in the south western extraplanar gas, its linewidth is a factor of 3 smaller than the observed one.

\subsection{Position-velocity diagram}

The position-velocity diagram along the major axis for the disk and extraplanar regions are shown in Fig. 9. The disk region is defined as the region between $-20^{\prime \prime}$ and $+11^{\prime \prime}$ from the major axis. The extraplanar region is defined as the region between $+11^{\prime \prime}$ and $+50^{\prime \prime}$ from the major axis. The following observed characteristics are reproduced by the model:

- the disk gas kinematics are relatively symmetric and normal, despite the strong north-south asymmetry of the gas distribution;

- the extraplanar gas has lower (blueshifted) velocities with respect to the disk gas.

On the other hand, our model does not reproduce the observed large linewidth (up to $150 \mathrm{~km} \mathrm{~s}^{-1}$ FWZI).

\section{MHD simulations}

\subsection{The model}

Otmianowska-Mazur \& Vollmer (2003) studied the evolution of the large scale magnetic field during a ram pressure stripping event. They calculated the magnetic field structure by solving the induction equation on the velocity fields produced by the dynamical model. The polarized radio continuum emission has been calculated by assuming a Gaussian spatial distribution of relativistic electrons. This procedure allowed them to study the evolution of the observable polarized radio continuum emission during a ram pressure stripping event.

We apply the same procedure as Otmianowska-Mazur \& Vollmer (2003) on a similar ram pressure stripping event (Sect. 2). The Zeus3D code (Stone \& Norman 1992a and b) is used to solve the induction equation:

$\partial \boldsymbol{B} / \partial t=\operatorname{rot}(\boldsymbol{v} \times \boldsymbol{B})-\operatorname{rot}(\eta \operatorname{rot} \boldsymbol{B})$

where $\boldsymbol{B}$ is the magnetic induction, $\boldsymbol{v}$ is the large-scale velocity of the gas, and $\eta$ is the coefficient of a turbulent diffusion. 

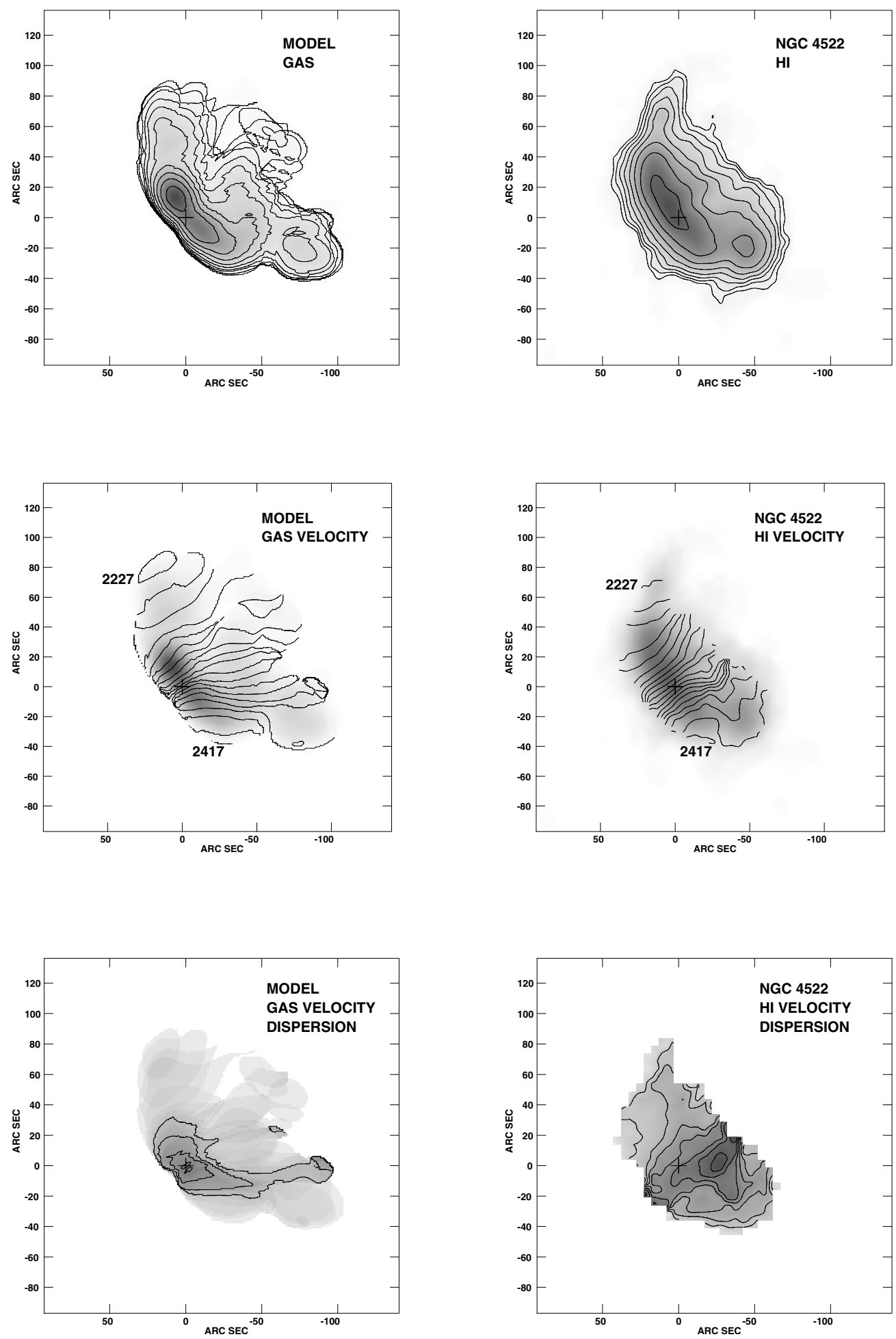

Fig. 8. Moment maps of NGC 4522. Left panels: model. Right panels: HI observations (Kenney et al. 2004). The same contouring scheme is used for both model and data. Upper panels: gas distribution; contours spaced by $\sqrt{2}$. Middle panel: velocity field; contours spaced $10 \mathrm{~km} \mathrm{~s}^{-1}$. Lower panel: velocity dispersion; contours: $15,20,25,30,35,40,45 \mathrm{~km} \mathrm{~s}^{-1}$.

We use a physical diffusion of $\eta=5 \times 10^{25} \mathrm{~cm}^{2} \mathrm{~s}^{-1}$ (Elstner et al. 2000). The estimated numerical diffusion is an order of magnitude smaller and thus does not affect our simulations. We do not use an $\alpha$ dynamo. The initial magnetic field is purely toroidal with a strength of $10 \mu \mathrm{G}$.

The induction equation is solved on a rectangular coordinates $(X Y Z)$. The number of grid points used is $171 \times 171 \times 71$ along the $X, Y$ and $Z$ axis, respectively. This corresponds to the grid spacing of $200 \mathrm{pc}$ in the galactic plane and of $300 \mathrm{pc}$ in the $Z$ direction, resulting in a size of the modeled box of $34.2 \mathrm{kpc} \times 34.2 \mathrm{kpc} \times 21.3 \mathrm{kpc}$. Since the $N$-body code is discrete whereas the MHD code is using a grid, we have to interpolate the discrete velocities on the grid. This is done using a method known as "Kriging" with a density-dependent smoothing length (Isaaks \& Srivastava 1989). It turned out that we had to use a large smoothing length to suppress the noise in the 

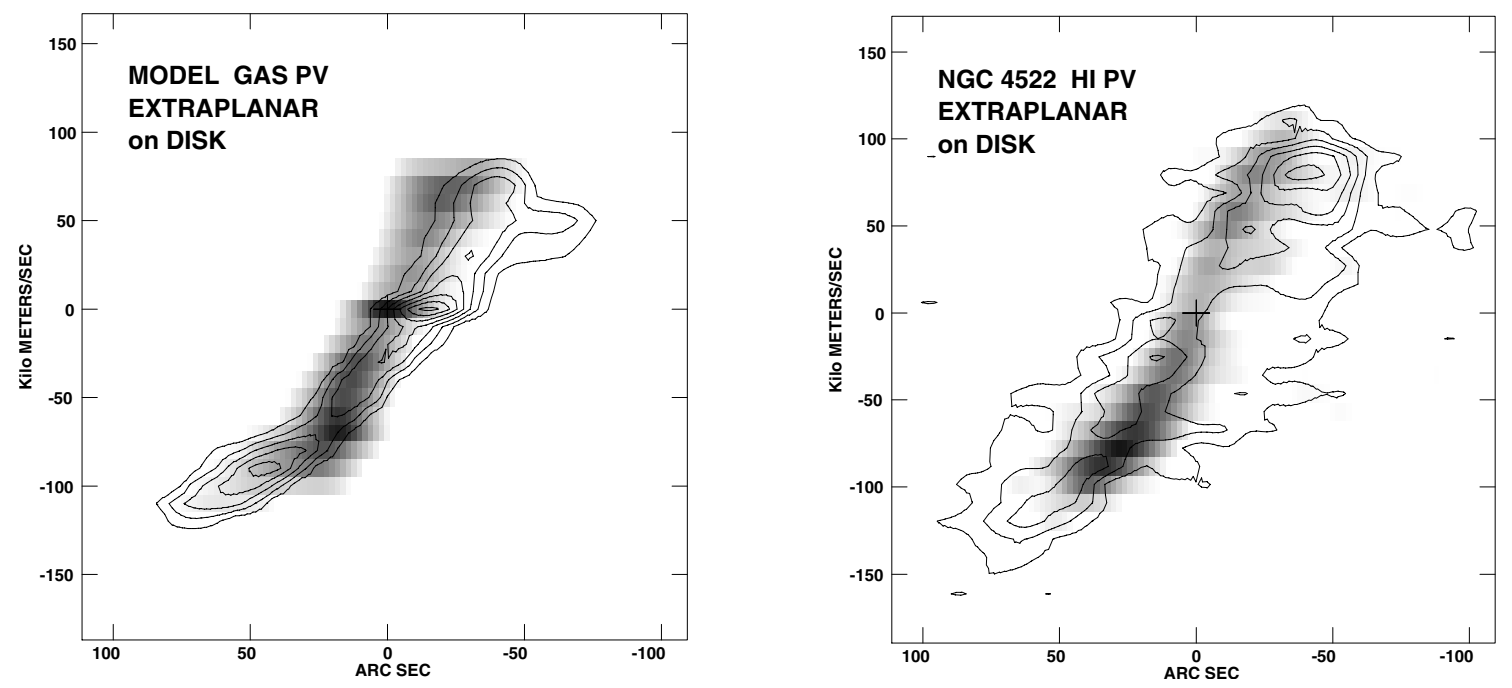

Fig. 9. Major axis position-velocity diagram for the disk (greyscale) and extraplanar (contours) component. Left panel: model. Right panel: HI observations (Kenney et al. 2004). Contour levels are 1, 2, 3, 4, 5, 6 times the peak value.

velocity field of the outer disk, which is due to a small, local particle density. In this way we avoid numerical artifacts of the magnetic field distribution at the outer disk. As the spline interpolation used in Otmianowska-Mazur \& Vollmer (2003), this has the consequence that the velocity field at the edge of the gas distribution is more extended than the gas distribution itself. Since there are gradients in this velocity field, induction leads to a magnetic field which extends beyond the edge of the gas distribution. This affects the polarized emission beyond the gas distribution, but not inside, which is what we are interested in.

The evolution of the polarized radio continuum emission without Faraday rotation is presented in Fig. 10. The timesteps are the same as in Fig. 5. The disk rotates clock-wise. The gas surface density, which is smoothed to a resolution of $\sim 100 \mathrm{pc}$, is shown in greyscales, the polarized radio continuum emission as contours, and the magnetic field vectors projected on the plane of the sky as lines. We assume a Gaussian distribution of relativistic electrons in $R$ and $z$ directions: $n_{\text {rel }}=n_{0} \exp \left(-\left(r / r_{R / z}\right)^{2}\right)$, where $r_{R}=5 \mathrm{kpc}$ and $r_{z}=0.5 \mathrm{kpc}$. This translates into a $F W H M$ of $8.3 \mathrm{kpc}$ and $1.2 \mathrm{kpc}$, respectively. By assuming this smooth distribution we imply no equipartition between the energy densities of total cosmic rays and total magnetic field. The galaxy moves to the lower left corner, i.e. the ram pressure wind comes from the this direction. The ram pressure maximum occurs at $t=0$ Myr. As already seen in Sect. 2 ram pressure begins to push the gas to the upper right corner at $t \sim-100$ Myr. The straight cut-off of the gas distribution in the upper right quadrant is due to the edge of our computational volume.

At $t>-100 \mathrm{Myr}$ the gas is compressed at the lower left side of the galaxy. At the same time most of the gas initially located in the outer disk $(R>3 \mathrm{kpc})$ is pushed out of the disk plane (Fig. 5). At $t>0$ Myr a prominent maximum of the polarized radio continuum emission forms in the lower left quadrant. Due to our large density-dependent smoothing length, a part of the largescale magnetic field in the lower left quadrant of the galaxy does not follow the compression and stays at large galactic radii in the disk plane (see also Otmianowska-Mazur \& Vollmer 2003). Therefore, the distribution of polarized intensity extends beyond that of the gas which is mostly due to our smoothing algorithm. However, a decoupling of the large-scale magnetic field from the gas flow is not entirely excluded (see e.g. Soida et al. 2001). Recently, Beck et al. (2005) could show that the regular magnetic field can decouple from the cold gas. The maximum of polarized emission within the gas disk in the lower left direction is real and due to gas compression. As observed in Otmianowska-Mazur \& Vollmer (2003) after maximum compression at $t=0$ Myr this inner maximum is taken along with rotation towards the upper left part of the image $(t>20 \mathrm{Myr})$. At $t=50 \mathrm{Myr}$ it is located to the left of the galaxy center, at $t=90 \mathrm{Myr}$ it is located on the upper left part of the gas disk. Since the FWHM of the relativistic electron distribution in the vertical direction is $1.2 \mathrm{kpc}$, the magnetic field taken away vertically with the gas is no longer observable in polarized radio continuum emission because of the low density of relativistic electrons there.

The evolution of the polarized radio continuum emission (Fig. 10) is qualitatively similar to that of Otmianowska-Mazur \& Vollmer (2003), where an inclination angle between the disk and the ICM wind direction of $i=20^{\circ}$ was assumed. Thus, the Otmianowska-Mazur \& Vollmer (2003) simulation corresponded to a more edge-on stripping, whereas in our simulation ram pressure stripping occurs more face-on $\left(i=60^{\circ}\right)$. Consequently, the gas compression is less pronounced in the present case. However, as in the Otmianowska-Mazur \& Vollmer (2003) simulations we observe that shortly after the timestep of maximum ram pressure the maximum of polarized radio continuum emission within the compressed gas disk is moving to the upper part of the image due to galactic rotation. However, ram pressure is still quite high and the ISM is still leaving the galactic disk at $t=50 \mathrm{Myr}$. This is preferentially happening on the side where galactic rotation and the ICM wind are parallel, i.e. in the north east.

As a conclusion, the polarized radio continuum emission outside the gas disk at timesteps $t>-20 \mathrm{Myr}$ is most probably due to our large density-dependent smoothing length due to the intrinsic difficulties of interpolating and extrapolating the 3D velocity field over a sharp edge of the gas distribution where no particles are found beyond a certain radius. In this case an interpolation and extrapolation with a density dependent smoothing length can always give rise to artifical velocity gradients which lead to an enhancement of the magnetic field. The here employed "Kriging" method with a density dependend smoothing length turned out to be a good compromise between the need of a smooth velocity and a minimum of artificial velocity gradients at the edge of the gas distribution. 

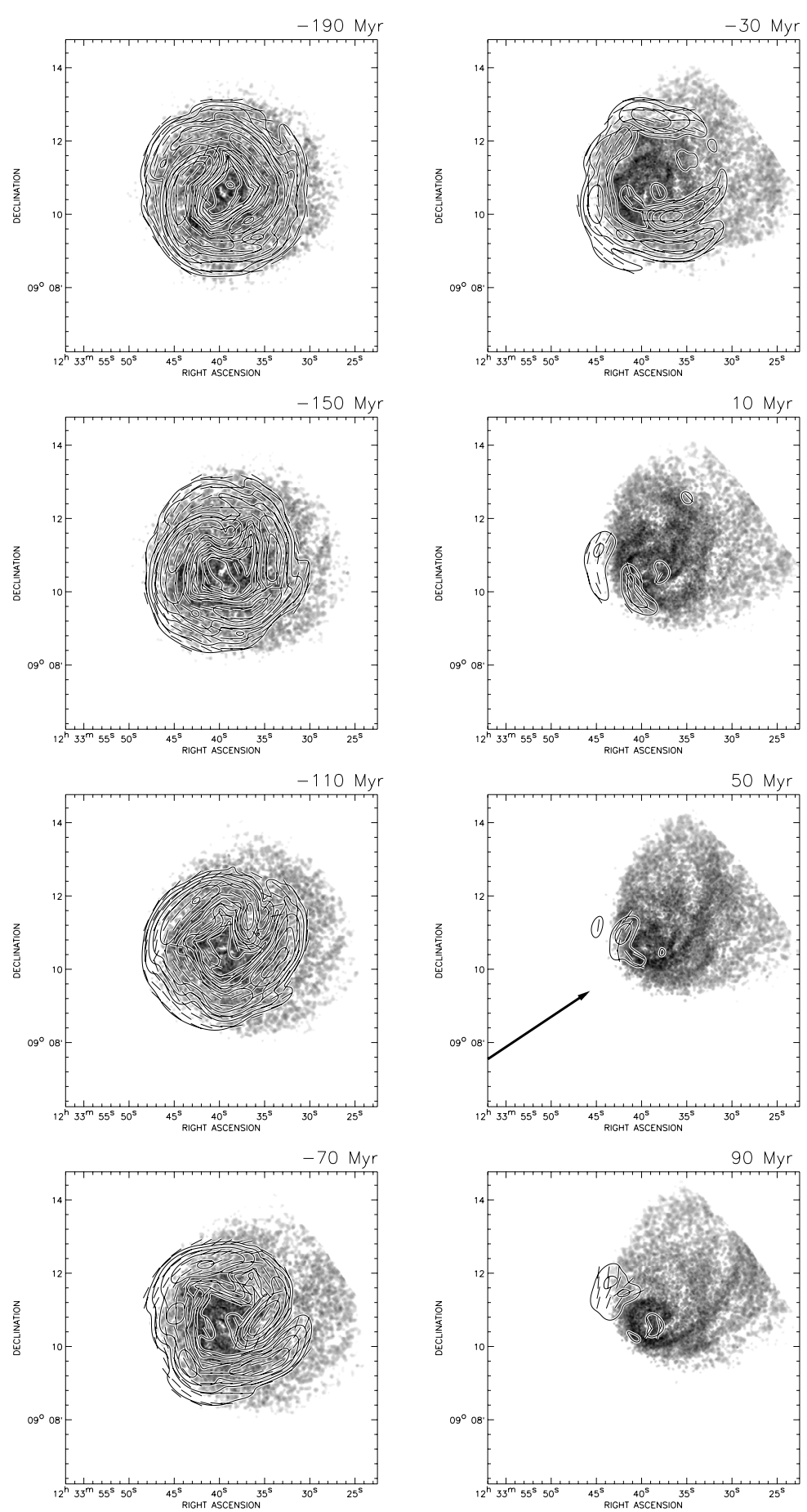

Fig. 10. Evolution of the polarized radio continuum emission. Time steps are the same as in Fig. 5. The disk is seen face-on and rotates clockwise. Contours: polarized radio continuum emission. The magnetic field vectors are superimposed onto the gas surface density. The timestep is indicated on top of each snapshot. The assumed beamsize for the polarized radio continuum emission is $20^{\prime \prime}$. The arrow in the last panel indicates the direction of the ram pressure wind.

However, the maximum of polarized radio continuum emission inside the compressed gas disk is real. Since we know the cause of polarized emission extending beyond the gas disk and since this feature and the real inner maximum of polarized emission are very close in space and since we did not want to modify the model by hand with a risk of introducing uncontrollable artifacts, we did not attempt to remove the part of the largescale magnetic field extending beyond the gas disk. We interpret the observed maximum of polarized radio continuum emission within the gas disk as being due to gas compression.

\subsection{Comparison with VLA polarized radio continuum emission}

The distribution of polarized radio continuum emission is now projected using the position and inclination angles of NGC 4522, $t=50 \mathrm{Myr}$, and the azimuthal angle as in Sect. 2.

The resulting model polarized radio continuum emission distribution and the model magnetic field vectors on the model gas distribution are shown in Fig. 11 together with our $6 \mathrm{~cm}$ VLA observations (Vollmer et al. 2004). As our observations, the model 
MODEL

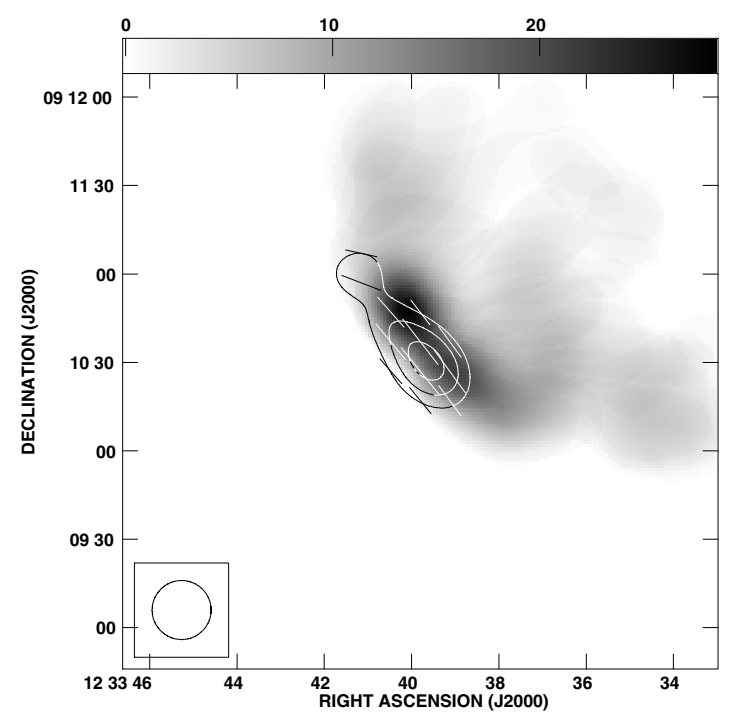

OBSERVATIONS

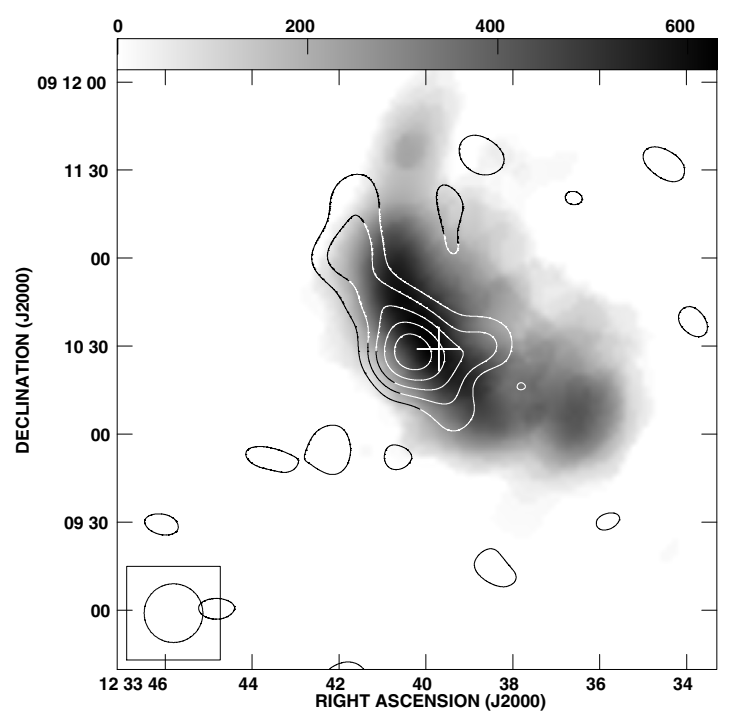

Fig. 11. Greyscale: HI gas distribution with a resolution of $\sim 20^{\prime \prime}$. Contours: polarized radio continuum emission. Left panel: model. The FWHM of the relativistic electron distribution in the radial and vertical directions are $8.3 \mathrm{kpc}$ and $1.2 \mathrm{kpc}$, respectively. Right panel: observations (Vollmer et al. 2004).

shows a polarized radio continuum maximum to the east of the galaxy center and a weaker extension to the north. The observed offset of the polarized emission maximum to the east translates the fact that the inclination angle of the galaxy is such that the eastern side of the disk is the compressed side which is the far side of the galaxy. Since the polarized emission extending beyond the edge of the gas distribution and the real compressed large-scale magnetic field are contiguous, the shown distribution of polarized radio continuum emission reflects that of the compressed component. The observed elongation of the polarized radio continuum emission can be explained as follows: due to gas compression a polarized emission maximum is created in the south-east. Shortly after the occurrence of the ram pressure maximum, the large-scale magnetic field follows rotation to the north-east. Thus, the observed polarized radio continuum emission corroborates our previous finding (based on the comparison between the model and the HI data) that we observe NGC 4522 after the occurrence of maximum ram pressure (Sect. 3 ).

\section{Discussion}

\subsection{Stripping of the cold and warm $\mathrm{HI}$}

For face-on stripping, our simulations are consistent with the Gunn \& Gott (1972) ram pressure estimate. Our dynamical model reproduces qualitatively and quantitatively the observed HI gas distribution, velocity field (Fig. 8) and polarized radio continuum emission distribution (Fig. 11). However, it fails in reproducing the observed large HI linewidths in the extraplanar component (Fig. 9). This is most probably due to a lack of gas physics in the model. The large observed linewidth of $\sim 100 \mathrm{~km} \mathrm{~s}^{-1}$ cannot be thermal, because this would translate into temperatures where the gas is ionized. Thus, they are due to directed, chaotic or turbulent gas motions. Since the HI line profiles tend to smaller velocities, i.e. to velocities closer to that of the Virgo cluster, one possible explanation is that a part of the atomic gas is stripped more efficiently than the rest. Since the column density of the low velocity gas is small, we speculate that it is a low density warm $(T \sim 8000 \mathrm{~K})$ atomic gas phase which is stripped more efficiently. In this scenario the large linewidth is due to directed motions. In a second scenario ram pressure leads to shocks in the interstellar medium which increase their chaotic or turbulent velocity dispersion dramatically. Again, as the column density of the low velocity gas is small, this might only concern the low density warm component of the atomic gas which has a larger volume filling factor than the cold atomic gas $(T \sim 100 \mathrm{~K})$. Both scenarios can explain the observed large HI linewidths in the extraplanar component. In both scenarios it is the warm diffuse atomic gas phase which produces the large linewidths.

\subsection{The stripping efficiency}

As already mentioned in Sect. 2 our knowledge about the orbit of NGC 4522 within the Virgo intracluster medium is poor. Its high radial velocity with respect to the cluster mean and its large distance from the cluster center exclude a simple radial orbit. Kenney et al. (2004) estimated ram pressure due to a static intracluster medium at the projected location of NGC 4522. Applying the Gunn \& Gott (1972) criterion and assuming an ICM density of $n_{\mathrm{ICM}}=10^{-4} \mathrm{~cm}^{-3}$ and a galaxy velocity of $1500 \mathrm{~km} \mathrm{~s}^{-1}$, they found that the gravitational restoring force for a gas cloud with a surface density of $10 \mathrm{M}_{\odot} \mathrm{pc}^{-2}$ is an order of magnitude higher than the force due to ram pressure. This led them to the conclusion that ram pressure is higher than the "standard value". This could in principle be due to a higher galaxy velocity or a higher stripping efficiency $\xi$ with $p_{\text {ram }}=\xi \rho\left(\boldsymbol{v}_{\text {gal }}-\boldsymbol{v}_{\mathrm{ICM}}\right)^{2}$ (see e.g. Roediger \& Hensler 2005).

Our simulation yields a projected ICM wind direction to the north east which is roughly aligned with the galaxy's minor axis $\left(6^{\circ} \pm 20^{\circ}\right.$; Fig. 5). This is corroborated by the recent results of Kenney et al. (in prep.) analysing dust extinction features in deep HST images of NGC 4522. They found gas clouds beyond the stripping radius in the direction of the ICM wind. Beyond the main gas/dust truncation radius, they found several elongated dust clouds, all with similar position angles of $-17^{\circ} \pm 3^{\circ}$ with respect to the minor axis, likely indicating the projected ICM wind direction. Moreover, our simulations yield an inclination angle between the disk and the ICM wind direction of $i=60^{\circ} \pm 10^{\circ}$ 
(more face-on stripping). Thus the wind angle is relatively faceon despite the fact that the galaxy us nearly edge-on and has a high line-of-sight velocity, implying that there must be a large component of the ICM wind in the plane of the sky. Based on our model the total velocity of the galaxy relative to a static ICM is $\sim 3500 \mathrm{~km} \mathrm{~s}^{-1}$ (see Sect. 3). With this velocity the Gunn \& Gott criterion is approximately fulfilled and the stripping efficiency is close to one.

\subsection{Static or moving ICM}

The comparison between HI observations and our model (Sect. 4) suggests that ram pressure acting on NGC 4522 is close to its maximum but has already passed it. The comparison between the observed and modeled polarized radio continuum emission confirms that the maximum has occurred recently ( $\sim 50$ Myr ago), because the polarized radio continuum emission extends to the north-east, in the direction of galactic rotation.

Thus, if the galaxy is crossing the cluster with a very high velocity $\left(\sim 4000 \mathrm{~km}^{-1}\right)$ and the ICM is static and smooth, NGC 4522 has just passed the point of highest intracluster medium density, i.e. it has just passed the point of smallest distance to M 87. On the other hand, if the intracluster medium is moving due to the infall of the M 49 group of galaxies as suggested by Kenney et al. (2004) and Vollmer et al. (2004), the galaxy has just passed the region of highest intracluster medium velocities. In this case ram pressure is given by Eq. (2). If the main component of $\boldsymbol{v}_{\text {gal }}$ is radial and thus $v_{\text {gal }} \sim 1500 \mathrm{~km} \mathrm{~s}^{-1}$, the ICM velocity vector $\boldsymbol{v}_{\mathrm{ICM}}$ must have dominant components in the plane of the sky. In the following we give an example:

We define the signs of velocity vectors as positive to the west, north, and away from the observer. Assuming a galaxy velocity vector of $\boldsymbol{v}_{\text {gal }}=(-675,-675,1155) \mathrm{km} \mathrm{s}^{-1}$ and taking the total velocity vector from our simulation $\boldsymbol{v}_{\mathrm{tot}}=\boldsymbol{v}_{\mathrm{gal}}-\boldsymbol{v}_{\mathrm{ICM}}=$ $(-2560,-2090,1155) \mathrm{km} \mathrm{s}^{-1}$ leads to a galaxy velocity of $v_{\mathrm{gal}}=$ $1500 \mathrm{~km} \mathrm{~s}^{-1}$ and $v_{\text {tot }}=3500 \mathrm{~km} \mathrm{~s}^{-1}$ (see Sect. 3). The resulting intracluster medium velocity is then $\boldsymbol{v}_{\mathrm{ICM}}=\boldsymbol{v}_{\mathrm{gal}}-\boldsymbol{v}_{\mathrm{tot}}=$ $(1885,1415,0) \mathrm{km} \mathrm{s}^{-1}$ and the total ICM velocity is $v_{\mathrm{ICM}}=$ $2360 \mathrm{~km} \mathrm{~s}^{-1}$. Thus the motion of the intracluster medium which might be partly due to the infall of the M49 subgroup is towards the north west and exclusively in the plane of the sky. This ICM velocity is $1000 \mathrm{~km} \mathrm{~s}^{-1}$ higher than that derived from X-ray data assuming pressure equilibrium (Shibata et al. 2001). Alternatively, a local ICM density enhancement of a factor $\sim 4$ together with an ICM velocity of $\sim 1000 \mathrm{~km} \mathrm{~s}^{-1}$ can account for the observed stripping radius of NGC 4522. This is consistent with the radial velocity of M $49\left(\sim 1000 \mathrm{~km} \mathrm{~s}^{-1}\right)$ which is very close to the velocity of the Virgo cluster. The derived direction of this putative ICM flow might also be related to the X-ray detection of a gas compression region in the north of M 49 (Irwin \& Sarazin 1996; Biller et al. 2005).

\section{Conclusions}

We present a dynamical model for the evolution of the interstellar medium of the Virgo cluster spiral galaxy NGC 4522, which presently undergoes strong ram pressure stripping (Kenney et al. 2004). We confront the observed with the simulated moment maps. In addition, we solve the induction equation on the velocity fields of the dynamical model to calculate the large-scale magnetic field. Assuming a smooth relativistic electron distribution we obtain the model distribution of polarized radio continuum emission which can be directly compared to observations (Vollmer et al. 2004).

We conclude that

- the model successfully reproduces the observed HI gas distribution and velocity field;

- the model fails in reproducing the observed large HI linewidths $\left(\sim 100 \mathrm{~km} \mathrm{~s}^{-1}\right)$;

- the MHD model successfully reproduces the observed polarized radio continuum emission;

We suggest that large observed HI linewidths is due to the warm diffuse component of the atomic gas which is either stripped more efficiently or whose velocity dispersion is increased by ram pressure induced shocks.

The model confirms that NGC 4522 undergoes ram pressure which is close to its maximum. This maximum ram pressure of $\sim 2000 \mathrm{~cm}^{-3}\left(\mathrm{~km} \mathrm{~s}^{-1}\right)^{2}$ occurred about $50 \mathrm{Myr}$ ago. The inclination angle between the galaxy's disk and the ICM wind direction is $i=60^{\circ} \pm 10^{\circ}$ and the ICM wind blows from the south-east. The projected wind angle with respect to the galaxy's minor axis is $6^{\circ} \pm 20^{\circ}$. Since the galaxy is located at a projected distance of $\sim 1 \mathrm{Mpc}$ from the cluster center where the intracluster medium density is by far too low to cause significant stripping if it is static, NGC 4522 is either on an unbound orbit within the cluster and just passed the region of highest Virgo intracluster medium density or, if the intracluster medium is moving, the galaxy just leaves the region of highest intracluster medium velocities. If the intracluster medium is static and smooth the galaxy has a total velocity of about $3500 \mathrm{~km} \mathrm{~s}^{-1}$ with respect to the Virgo cluster. If the intracluster medium is moving possibly due to the infall of the M49 group of galaxies, we derive a velocity of the infalling intracluster medium of about $2400 \mathrm{~km} \mathrm{~s}^{-1}$ with respect to the Virgo cluster. In this case the intracluster medium associated with the M 49 group is moving towards the north east with a negligible radial velocity component.

This study shows the strength of combining high resolution HI and polarized radio continuum emission with detailed numerical modeling of the evolution of the gas and the large-scale magnetic field.

Acknowledgements. This work was supported by Polish-French (ASTROLEA-PF) cooperation program, and by Polish Ministry of Sciences grant PB $378 / \mathrm{P} 03 / 28 / 2005$

\section{References}

Beck, R., Fletcher, A., Shukurov, A., et al. 2005, A\&A, 444, 739

Biller, B. A., Jones, C., Forman, W. R., Kraft, R., \& Ensslin, T. 2004, ApJ, 613, 238

Boulares, A., \& Cox, D. P. 1990, ApJ, 365, 544

Elmegreen, B. G., \& Falgaron, E. 1996, ApJ, 471, 816

Elstner, D., Otmianowska-Mazur, K., von Linden, S., \& Urbanik, M. 2000, A\&A, 357, 129

Irwin, J. A., \& Sarazin, C. L. 1996, ApJ, 471, 683

Isaaks, E. H., \& Srivastava, R. 1989, An Introduction to Applied Geostatistics (Oxford University Press), Chap. 12

Kenney, J. P. D., \& Koopmann, R. A. 1999, AJ, 117, 181

Kenney, J. P. D., van Gorkom, J., \& Vollmer, B. 2004, AJ, 127, 3361

Otmianowska-Mazur, K., \& Vollmer, B. 2003, A\&A, 402, 879

Roediger, E., \& Hensler, G. 2005, A\&A, 433, 875

Shibata, R., Matsushita, K., Yamasaki, N. Y., et al. 2001, ApJ, 549, 228

Soida, M., Urbanik, M., Beck, R., Wielebinski, R., \& Balkowski, C. 2001, A\&A, 378, 40

Springel, V., Yoshida, N., \& White, S. D. M. 2001, NewA, 6, 79

Stone, J. M., \& Norman, M. L. 1992a, ApJS, 80, 791

Stone, J. M., \& Norman, M. L. 1992b, ApJS, 80, 791

Vollmer, B., Cayatte, V., Balkowski, C., \& Duschl, W. J. 2001, ApJ, 561, 708

Vollmer, B., Beck, R., Kenney, J. P. D., \& van Gorkom, J. H. 2004, AJ, 127, 3375

Wiegel, W. 1994, Diploma Thesis, University of Heidelberg 